\title{
Erratum: Magnetic Moment Manipulation by a Josephson Current [Phys. Rev. Lett. 102, 017001 (2009)]
}

\author{
F. Konschelle and A. Buzdin (1)
}

(Received 19 September 2019; published 17 October 2019)

DOI: 10.1103/PhysRevLett.123.169901

There was an error in the sign of the vector product of $\mathbf{M}$ and $\mathbf{H}_{\mathrm{eff}}$ in the Landau-Lifshitz-Gilbert equation, Eq. (6) in the original Letter, which effectively corresponds to the sign change of gyromagnetic ratio $\gamma$. Therefore the presented results correspond simply to the inverted direction of the spin precession. This does not affect the qualitative conclusions of the Letter but leads to some sign changes in the formulas.

Equation (9) should read

$$
m_{x}=\frac{\Gamma \omega \cos \omega t}{1-\omega^{2}} \quad \text { and } \quad m_{y}=\frac{\Gamma \sin \omega t}{1-\omega^{2}}
$$

Equation (11) should read

$$
m_{y}(t)=\frac{\omega_{+}-\omega_{-}}{r} \sin \omega t-\frac{\alpha_{+}+\alpha_{-}}{r} \cos \omega t .
$$

Expression (12) should read

$$
\omega_{ \pm}=\frac{\Gamma r}{2} \frac{\omega \pm 1}{\Omega_{ \pm}} \quad \text { and } \quad \alpha_{ \pm}=\frac{\Gamma r}{2} \frac{\alpha \omega}{\Omega_{ \pm}}
$$

with $\Omega_{ \pm}=(\omega \pm 1)^{2}+\alpha^{2} \omega^{2}$.
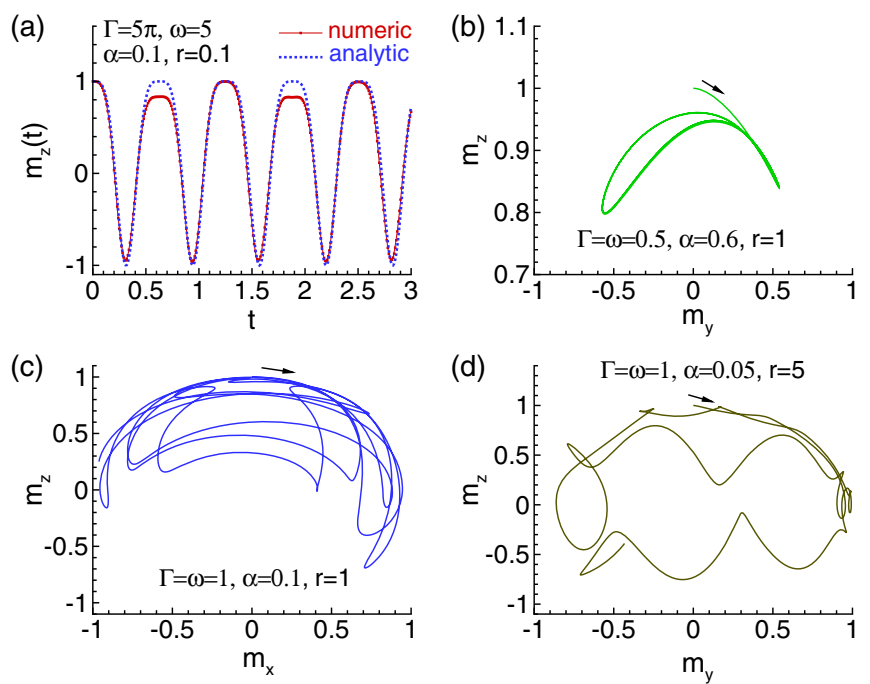

FIG. 2. Results of numerical analysis of the magnetic moment dynamics of the $\varphi_{0}$-junction. (a) Reversal of $m_{z}$ from analytical expression Eq. (15) (dashed curve) and numerical one (normal curve). The other curves are related to the M trajectory: (b) in strong damping case (c) and (d) in the strong coupling regime revealing incomplete and complete magnetic moment reversal, respectively. 
Equation (13) should read

$$
I(t)=I_{c} \sin \omega t-I_{c} \frac{\omega_{+}-\omega_{-}}{2} \sin 2 \omega t+I_{c} \frac{\alpha_{+}+\alpha_{-}}{2} \cos 2 \omega t+I_{0}(\alpha) .
$$

Equation (14) should read

$$
I_{0}(\alpha)=I_{c} \frac{\alpha \Gamma r \omega}{4}\left(\frac{1}{\Omega_{-}}+\frac{1}{\Omega_{+}}\right)
$$

The results of numerical analysis of the magnetic moment dynamics for the parameters used in Fig. 2 have been updated.

We are indebted to Yu. Shukrinov and I. Rahmonov for pointing out the errors and assistance in performing the numerical calculations. 